\title{
Bazex-syndrome with esophageal cancer
}

\section{Sebastian Boldt, Uwe Wollina}

Department of Dermatology and Allergology, Academic Teaching Hospital Dresden-Friedrichstadt, 01067 Dresden, Germany

Corresponding author: Prof. Uwe Wollina, E-mail: wollina-uw@khdf.de

\begin{abstract}
Skin diseases can be a first sign of internal malignancies. Acrokeratosis neoplastica or Bazex-syndrome is a rare obligatory paraneoplastic dermatosis. We report on a 51-year-old male patient who complaint about reduced physical working capacity, swelling of fingers and toes. He presented with non-itching violaceous erythemato-squamous lesions on nose, hands and feet. On examination we observed onychomadesis of finger nails. Thoracic computerized tomography disclosed a tumor mass in the distal esophagus. Endosonography with trans-esophageal fine-needle biopsy confirmed the diagnosis of esophageal squamous cell carcinoma. The knowledge of cutaneous paraneoplasias allows an earlier diagnosis of internal malignancies.
\end{abstract}

Key words: Paraneoplastic skin disease; Acrokeratosis neoplastica; Bazex-syndrome; Esophageal squamous cell carcinoma

\section{INTRODUCTION}

Human skin can be a mirror for internal disorders including malignancies. The cutaneous signs and symptoms may often precede the development of clinical tumor-associated complaints. The so-called cutaneous paraneoplasias will help to identify internal malignancies earlier [1].

We differentiate cutaneous paraneoplasias in facultative such as erythema gyratum repens and obligate such as acrokeratosis paraneoplastica. The latter is also known as Bazex-syndrome. Bazex et al. described the syndrome in 1965 in a patient with a larynx tumor [2]. In 75\% of cases Bazex-syndrome develops before the diagnosis of malignancies. Its diagnosis should lead to a detailed search for cancer [3]. Associated tumors are mainly found in pharynx and larynx (48.6\%), lungs (17.7\%), and esophagus (10.6\%) [2].

Here we report a 51-year-old male patient with Bazexsyndrome and esophageal squamous cell carcinoma (SCC).

\section{CASE REPORT}

A 51-year-old male patient was referred from the rheumatologist for diagnosis of cutaneous lesions, that developed during the last 5 months. He had a medical history for arterial hypertension, chronic obstructive pulmonary disease (COPD), and smoking. His medical family history was unremarkable.

He noticed a reduced physical working capacity. The patient also complaint about swelling of fingers and toes. He had neither arthritis nor arthralgia or morning stiffness, no dysphagia. Skin of nose, hands and feet presented with violaceous erythema and scaling (Fig. la and lb). The lesions were not itching. Fingernails demonstrated onychomadesis (Fig. 2).

Laboratory investigations were unremarkable for routine lab, antinuclear antibodies, antibodies against extractable nuclear antigens, citrullin, rheumatoid factor. Neither cryoglobulins nor cold agglutinins were detected. Immune fixation was normal. Only minor iron-deficient anemia was noted.

He had no signs of vascular disorders. 


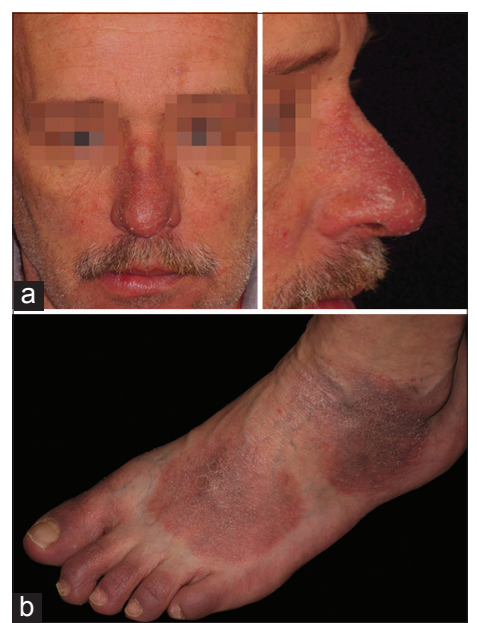

Figure 1: Acrokeratosis neoplastica (Bazex-syndrome). Violaceous erythemato-squamous lesions resembling psoriasis. (a) Nose. (b) Feet with edematous swelling of toes.

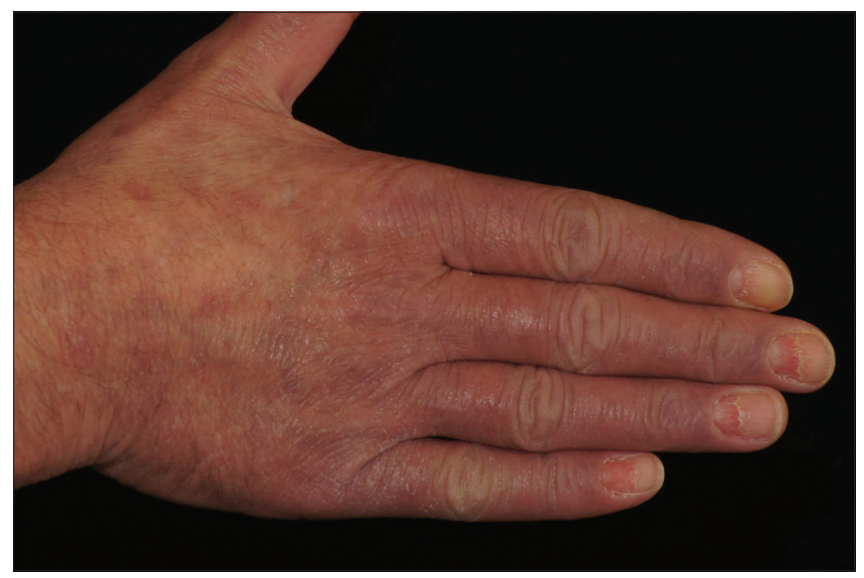

Figure 2: Hand in Bazex-syndrome with psoriasis-like cutaneous lesions and onychomadesis of nails.

Computerized tomography (CT) disclosed a tumor mass of the esophagus measuring about $36 \times 39 \mathrm{~mm}$ (Figs. $3 a$ and $3 b$ ). Endosonography with transesophageal fine-needle puncture was performed to obtain tissue for histopathology. The diagnosis of a squamous cell carcinoma (SCC) of the esophagus was confirmed.

Palliative chemotherapy or radiotherapy were suggested since the tumor could no longer been resected surgically.

\section{DISCUSSION}

Acrokeratosis neoplastica or Bazex-syndrome is a rare obligate paraneoplasia. The typical patient is older than 50 years of age. There is a male predominance. Tumors of upper airways and digestive tract are on top of malignancies associated with Bazex-syndrome [2-7].

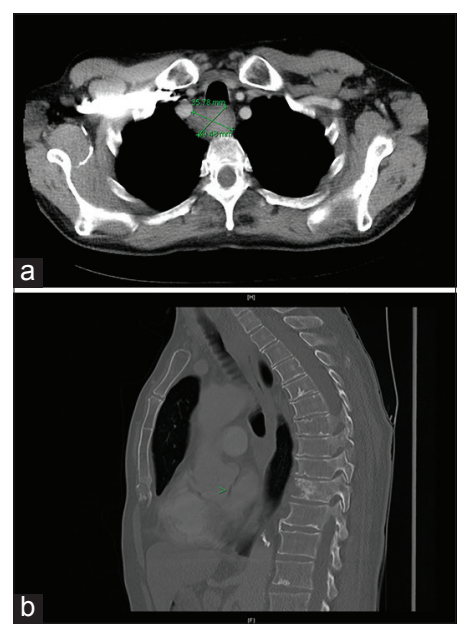

Figue 3: Computerized tomography of esophageal squamous cell carcinoma. (a) Transaxial view and (b) sagittal view. The arrow in (a) points to the tumor.

Cutaneous symptoms resemble psoriasis with bluish or violaceous erythema and scaling, onychodystropy, and/ or keratoderma. There is usually no pruritus. Typically, these lesions occur symmetrical on acral body parts like ears, nose, hands and feet [2-5]. Uncommon manifestations include flexural Bazex-syndrome, which was seen in a patient with tonsillar adenocarcinoma [6], or genital involvement [7]. Histopathology of cutaneous lesions is non-specific psoriasiform.

Our patient demonstrated onychomadesis of finger nails like the patient of Graves et al [7]. Other nail findings are subungual keratosis, yellowish discoloration, ridging, and onycholysis [2].

We detected an associated esophageal SCC. The patient had not reported any symptoms. Due to the size and topography of the tumor curative surgery was, however, no longer possible. Radiotherapy with cumulative doses between 50 to 70 Gy leads to 5-year survival rates of $23 \%$ [8].

In contrast to tumor therapy, treatment of cutaneous Bazex symptoms is of minor importance. Moisturizers and topical corticosteroids may be used but are not very effective. On the other hand, Bazex-syndrome disappears in more than $90 \%$ after successful tumor therapy [9] but in other cases Bazex-syndrome persists due to unknown reasons [10].

\section{REFERENCES}

1. Silva JA, Mesquita Kde C, Igreja AC, Lucas IC, Freitas AF, Oliveira SM, Costa IM, Campbell IT. Paraneoplastic cutaneous 


\section{www.odermatol.com}

manifestations: concepts and updates. An Bras Dermatol. 2013;88:9-22.

2. Bazex A, Salvador R, Dupré A, Christol B. Syndrome paranéoplasique à type d'hyperkératose des extrémités. Guérison après le traitement de l'épithélioma laryngé. Bull Soc Franc Derm Syph. 1965;72:182.

3. Bolognia JL. Bazex syndrome: acrokeratosis paraneoplastica. Semin Dermatol. 1995;14:84-9.

4. Thiel W, Plog B, Schreiber G, Wollina U. Paraneoplastic acrokeratosis (Bazex syndrome). Hautarzt. 1987;38:304-7.

5. Humphrey SR, Hussain AS, Chandran R, Wilson B, George B. Acute onset of acrokeratosis paraneoplastica (Bazex syndrome). JAMA Dermatol. 2015;151:677-8.

6. Ali M, Keir M, Dodd H, Cerio R. Flexural Bazex syndrome associated with tonsillar adenocarcinoma. J Drugs Dermatol. 2004;3:557-9.

7. Graves MS, Hardin ME, Davis LS. A case of Bazex syndrome with genital involvement. JAMA Dermatol. 2014;150:1368-70.
8. Chen DF, Yang ZY, Yin WB. Radiotherapy of 180 cases of operable esophageal carcinoma. World J Gastroenterol. 1997;3:123-6.

9. Dourmishev LA, Draganov PV. Paraneoplastic dermatological manifestation of gastrointestinal malignancies. World J Gastroenterol. 2009;15:4372-9.

10. Fasanmade A, Farrell K, Perkins CS. Bazex syndrome (acrokeratosis paraneoplastica): persistence of cutaneous lesions after successful treatment of an associated oropharyngeal neoplasm. Br J Oral Maxillofac Surg. 2009;47:138-9.

Copyright by Sebastian Boldt, et al. This is an open access article distributed under the terms of the Creative Commons Attribution License, which permits unrestricted use, distribution, and reproduction in any medium, provided the original author and source are credited.

Source of Support: Nil, Conflict of Interest: None declared. 